\title{
Electronic Health Record System-Related Patient Safety Incidents - How to Classify Them?
}

\author{
Sari PALOJOKI ${ }^{\mathrm{a}, 1}$, Riikka VUOKKO ${ }^{\mathrm{a}}$, Anne VAKKURI ${ }^{\mathrm{b}}$ and Kaija SARANTO ${ }^{\mathrm{c}}$ \\ ${ }^{a}$ The Ministry of Social Affairs and Health, Finland \\ ${ }^{b}$ Helsinki University Hospital (HUS), Peijas Hospital, Finland \\ ${ }^{\mathrm{c}}$ University of Eastern Finland, Finland
}

\begin{abstract}
The implementation of electronic health record systems (EHRs) may cause multidimensional patient safety issues that deserve research attention. Our research aims to identify the current body of evidence on EHRs-related incident types and how incidents are classified in these studies. A literature search resulted in 44 peer-reviewed papers and six papers were included in the final analysis. The error types do not concern solely the technological features of the EHRs but may involve also non-technical aspects. Our review indicates that standard classification systems would facilitate comparisons across countries. To achieve the goal, more research evidence, testing and development of classifications are required.
\end{abstract}

Keywords. Patient safety, incident reporting, electronic health record system, error, classification

\section{Introduction}

Health and biomedical informatics communities have long been interested in unintended consequences arising from the implementation of electronic health record systems (EHRs). While EHRs may enhance the safety of patient care, it is also assumed that an increase in the implementation of information technology within healthcare systems will lead to patient safety incidents by introducing novel vulnerabilities and unique risks. Even if the body of research identifying technology-induced errors related to EHRs is growing, there is a lack of risk reporting, and data describing those risks is still limited. [1-4]

There is a growing body of evidence pointing to several methods that can be used to address technology-induced errors. Patient safety incident reporting by end-users is the primary mechanism by which it is possible to learn about these concerns [3-5]. The European Council [6] recommends that Member States support blame-free reporting systems, which provide information about the extent, types, and causes of incidents. Incident reporting systems (IRS) have now been in place for more than a decade in many countries but it is not well established how to define and classify events in these systems. Moreover, comprehending the limitations of patient safety incident data is indispensable in avoiding the misinterpretation of the data $[5,7]$.

\footnotetext{
${ }^{1}$ Corresponding author, Dr. Sari Palojoki, sari.palojoki@stm.fi
} 
EHR concerns as a multidimensional patient safety issue deserve research attention. Analysis of patient safety incidents produces useful data [3-4, 14]. Based on a literature review our research aims to identify the current body of evidence on EHRsrelated incident types based on classification systems. Our research questions are: (1) Which are the most common types of electronic health record system-related patient safety incidents? 2) How EHRs-related patient safety incidents are classified in these studies?

\section{Methods}

In this paper, we apply a method for our literature review, which is consistent with guidelines by Templier and Paré [8]. Steps consist of formulating the research questions, searching the literature, screening for inclusion, extracting data, and analyzing data. Step "assessing the quality of primary studies" was excluded due to the research focus. Terms for literature searches in the PubMed database were composed according to appropriate MeSH terms "patient safety", "incident reporting", "voluntary incident reporting", "patient safety reporting", "electronic health record/system", "EHR/s", "information system" and "computer". The terms were grouped into sets and terms were combined with the "OR" operator, and all sets were then combined with the "AND" operator.

In this context, we conceptualize EHRs-related patient safety incidents as errors being realized in a complex healthcare environment during the use of EHRs [e.g. 5]. Research that focused on technology-induced errors associated with EHRs in connection with IRS were eligible for inclusion. Our search strategy covered the use of all types of EHRs in any type of clinical setting. Studies published in peer-reviewed journals or conference proceedings were included but editorials were excluded. Papers published in English without any restrictions of timeframe were included.

A literature search in the middle of July 2020 resulted in 44 peer-reviewed papers. After removing duplicates and the first exclusion round based on the researcher reading the abstracts, 12 papers were selected for further reading. Criteria for exclusion were the following: the language was not English and the research was out of scope, e.g. the focus was on incident reporting system development. After full paper reading, an additional seven of the research papers were excluded as they were out of scope. Additionally, one peer-reviewed article was retrieved based on full paper review [3]. A total of six papers was included in the final analysis based on our research questions.

\section{Results}

\subsection{Common Types of Electronic Health Record System-Related Patient Safety Incidents}

The results indicate that there is yet little evidence for research on types of EHRs-related patient safety incidents. Some studies reported underlying causes of incidents and mentioned e.g. failures related to communication with other care providers [9]. However, a more detailed EHRs-related analysis was not the scope of the study.

Magrabi et al. [10] performed a study, which examined a broader scope of computerrelated patient safety incidents. Computer-related patient safety incidents in a national AIMS database were analyzed. Only $0.2 \%$ of all reports in the database were computer- 
related. Machine-related problems were more common than human-computer interaction issues. However, they also found human-computer interaction errors related to the selection of patient and clinical information, as well as display errors.

A study on radiology systems revealed that communication breakdown was a contributing factor in $49 \%$ of incidents $(n=209)$ reported. An association with data collection, storage, or retrieval of electronic information was found in 147 of the 209 incidents. One-tenth of the incidents indicated that EHRs contributed to errors. [11]

Research on radiation oncology incident reports focused on potentially significant clinical consequences. Totally, $53 \%$ of events $(n=1507)$ with a potential high severity rating were related to human error. The most common human error reported concerned about the design of a suboptimal treatment plan. Almost one-third of events were related to an error at the level of the human-software interface, $2 \%$ were hardware failures, $1 \%$ were software failures, and $1 \%$ concerned an error in the software-hardware interface. Additionally, events rated with a maximum potential severity were related to a mismatch of information between the treatment planning software and the treatment management system and with manual data entry errors. [12]

Patient safety incidents, which included all aspects of IT within the healthcare context were studied in the UK. The majority of the reports $(77 \%)$ were machine-related technical problems, such as software errors, access, and display problems, and system downtime. A further $10 \%$ of the reports were related to human-computer interaction issues, and $13 \%$ of the incidents could not be classified using the framework. Only rare human error events were identified. [13]

EHRs-related safety issues reported within a voluntary reporting system by applying a sociotechnical conceptual model that included both technical and non-technical dimensions of safety. Non-technical dimensions, such as workflow, policies, and personnel, interacted frequently with technical dimensions, which included software/hardware, content, and user interface, to produce safety concerns. A total of $94 \%$ of incidents related to unmet data display needs in the EHR, data transmission problems and 'hidden dependencies' related to the EHR. [3]

EHRs-related patient safety incidents were analysed in an incident reporting database in hospitals with 100\% EHR implementation rate. Data from 23 hospitals during a 2-year period indicated that the proportion of electronic health record-related incidents was higher than in previous studies with similar data. Human-computer interaction problems were the most frequently reported. [14]

\subsection{Classification Systems of EHRs -Related Patient Safety Incidents}

Our research illustrates that classification development is documented in heterogeneous ways. For example, in Australia [10] work was carried out to identify natural categories for classification based on the incident data available. Based on previous research and analysis of incident reports, the Advanced Incident Management System (AIMS) classifies incidents to 13 incident types. It distinguishes human-computer interactionbased errors (e.g. wrong patient selected) from machine-related problems. After that, incidents were subdivided based upon problems at the point of data entry (input), data transfer (transfer), or data retrieval (output). A category of 'contributing factors' was also included to account for other socio-technical contextual variables that contributed to computer-related incidents (e.g. multi-tasking while using a computer).

Similarly, information technology-related incidents were analysed based on a Welsh voluntary IRS [13] to understand the implications of these incidents for 
healthcare. In the analysis, the AIMS classification was used. The results point out that the AIMS classification is dependent on the original data used to develop it, and thus, not all clinical relevance of the Welsh incidents could be captured with the classification. The research suggests that a different approach is needed to explore the clinical implications of incidents more appropriately. In a Dutch cohort study [9], the underlying causes of incidents were classified with three main classes (organizational, human, and patient-related), where the two first classes have several sub-classes. The research indicates that incomplete patient records increase the risk of incidents.

In the English National Health Service (NHS) context [3], an EHR implementation research with a patient safety focus was carried out by applying a sociotechnical model and a three-phase patient safety model (safe technology, safe use of technology, and use of technology to improve safety) to data from 12 NHS hospitals. Patient safety concerns were classified into eight main classes where each class had defined characteristics, continued with a review of risks and incidents related to each class with the professionals to both review the incident and to develop the model. Although the classification relates to risks of EHRs during the implementation phase, it may have the potential to inform safety risks.

The Finnish patient safety IRS (HaiPro) based classification was used to define safety incidents in hospitals with 100\% EHRs implementation rate [14]. Here, incidents are classified with 13 main classes and their sub-classes, of which the most frequently used main classes are 'Medication and Transfusions', 'Information Flow' and 'Information Management' categories as well as 'Laboratory', 'Imaging' and 'Other Patient Treatment Procedures' categories. The classification was built into the HaiPro system, and as such could not be modified by the incident reporter.

Research in an oncology setting [12] applied a French Nuclear Safety Authority (ASN) 5-point scale to classify events. ASN has seven classes: human, software and hardware errors, errors in communication between two humans, at the human-software interface, at the software-hardware interface, and at the human-hardware interface. The results indicate that the NRS could inform also other classification development.

\section{Discussion}

Researchers have developed ways of identifying and addressing types of errors in EHRs. Patient safety incident reporting systems (IRS) are an important part of safety programs, but the difficulty in analyzing error reports has limited their utility [4,5]. Research data on IRS is still scarce. In our review, the error types are not related solely to the technological features of the EHRs but may involve users of EHRs, their workflows, and aspects of the organizations in which they function. In summary, presumably, patient safety risks associated with EHRs vary along the adoption and implementation timeline of EHRs [see also, 3].

Our review indicates that the use of standard classifications would facilitate data use across countries. However, research notes that there is limited evidence of the development of investigative frameworks or classifications to categorize and comprehend the nature and e.g. clinical implications of the incidents [13]. There is a need to continually standardize the incident categories as well as train health professionals about how to report on types of EHRs-related errors [5]. For example, in EHRs, a medication administration error may have been due to missing data, but it is reported as a medication incident rather than a data capture event $[13,14]$. Moreover, narrative 
information and evidence-based classification development presented in research may serve as a basis for improving classifications and in turn, incident reporting.

Although IRS serves a purpose to corrective actions, attention has to be paid to the potential bias in reporting patterns that comes from uneven participation [12]. One of the limitations of these studies is that number of events that are reported is likely low. Reports do not provide exact frequencies of incidents but rather a descriptive analysis of EHRs-related safety problem types [14].

As a conclusion, there are only a little research results on EHRs-related error types. Classifications are potential tools and key enablers for the identification of incidents and for better use of data across countries. To achieve the goal, more research evidence and testing and development of existing classifications are needed.

\section{References}

[1] Kuziemsky CE, Randell R, Borycki EM. Understanding Unintended Consequences and Health Information Technology:. Contribution from the IMIA Organizational and Social Issues Working Group. Yearb Med Inform. 2016 Nov 10;(1):53-60. doi: 10.15265/IY-2016-027. PMID: 27830231; PMCID: PMC5171563.

[2] Denham CR, Classen DC, Swenson SJ, Henderson MJ, Zeltner T, Bates DW. Safe use of electronic health records and health information technology systems: trust but verify. J Patient Saf. 2013 Dec;9(4):177-89. doi: 10.1097/PTS.0b013e3182a8c2b2. PMID: 24257062.

[3] Meeks DW, Takian A, Sittig DF, Singh H, Barber N. Exploring the sociotechnical intersection of patient safety and electronic health record implementation. J Am Med Inform Assoc. 2014 Feb;21(e1):e28-34. doi: 10.1136/amiajnl-2013-001762. Epub 2013 Sep 19. PMID: 24052536; PMCID: PMC3957388.

[4] Palojoki S, Pajunen T, Saranto K, Lehtonen L. Electronic Health Record-Related Safety Concerns: A Cross-Sectional Survey of Electronic Health Record Users. JMIR Med Inform. 2016 May 6;4(2):e13. doi: 10.2196/medinform.5238. PMID: 27154599; PMCID: PMC4890731.

[5] Borycki E, Dexheimer JW, Hullin Lucay Cossio C, Gong Y, Jensen S, Kaipio J, Kennebeck S, Kirkendall E, Kushniruk AW, Kuziemsky C, Marcilly R, Röhrig R, Saranto K, Senathirajah Y, Weber J, Takeda H. Methods for Addressing Technology-induced Errors: The Current State. Yearb Med Inform. 2016 Nov 10;(1):30-40. doi: 10.15265/IY-2016-029. PMID: 27830228; PMCID: PMC5171580.

[6] The Council of the European Union. COUNCIL RECOMMENDATION of 9 June 2009 on patient safety, including the prevention and control of healthcare associated infections (2009/C 151/01). https://ec.europa.eu/jrc/sites/jrcsh/files/2_June_2009\%20patient\%20safety.pdf.

[7] Shojania KG, Marang-van de Mheen PJ. Temporal trends in patient safety in the Netherlands: reductions in preventable adverse events or the end of adverse events as a useful metric? BMJ Qual Saf. 2015 Sep;24(9):541-4. doi: 10.1136/bmjqs-2015-004461. Epub 2015 Jul 6. PMID: 26150549.

[8] Templier M and Pare G.A Framework for Guiding and Evaluation Literature Reviews. Communications of the Association for Information Systems 37(6):112-137. August 2015. DOI: 10.17705/1CAIS.03706.

[9] van Dulmen SA, Tacken MA, Staal JB, Gaal S, Wensing M, Nijhuis-van der Sanden MW. Patient safety in primary allied health care: what can we learn from incidents in a Dutch exploratory cohort study? Med Care. 2011 Dec;49(12):1089-96. doi: 10.1097/MLR.0b013e3182367105. PMID: 22002643.

[10] Magrabi F, Ong MS, Runciman W, Coiera E. An analysis of computer-related patient safety incidents to inform the development of a classification. J Am Med Inform Assoc. 2010 Nov-Dec;17(6):663-70. doi: 10.1136/jamia.2009.002444. PMID: 20962128; PMCID: PMC3000751.

[11] Stewart MJ, Georgiou A, Hordern A, Dimigen M, Westbrook JI. What do radiology incident reports reveal about in-hospital communication processes and the use of health information technology?. Stud Health Technol Inform. 2012;178:213-218.

[12] Terezakis SA, Harris KM, Ford E, Michalski J, DeWeese T, Santanam L, Mutic S, Gay H. An evaluation of departmental radiation oncology incident reports: anticipating a national reporting system. Int J Radiat Oncol Biol Phys. 2013 Mar 15;85(4):919-23. doi: 10.1016/j.ijrobp.2012.09.013. Epub 2012 Dec 19. PMID: 23265568.

[13] Warm D, Edwards P. Classifying health information technology patient safety related incidents - an approach used in Wales. Appl Clin Inform. 2012 Jun 27;3(2):248-57. doi: 10.4338/ACI-2012-03-RA0010. PMID: 23646074; PMCID: PMC3613022.

[14] Palojoki S, Mäkelä M, Lehtonen L, Saranto K. An analysis of electronic health record-related patient safety incidents. Health Informatics J. 2017;23(2):134-145. doi:10.1177/1460458216631072. 